\title{
UV-Metric, pH-Metric and RP-HPLC Methods to Evaluate the Multiple pKa Values of a Polyprotic Basic Novel Antimalarial Drug Lead, Cyclen Bisquinoline
}

Mohammad Faisal Hossain, Cassandra Obi, Anjuli Shrestha and MO Faruk Khan*

SCRiPS, College of Pharmacy, Southwestern Oklahoma State University, USA

\begin{abstract}
The purpose of this experiment was to evaluate and compare the pKa values of the poorly water soluble, weakly basic, novel antimalarial drug lead, 4,10-bis (7-chloroquinoline)-1,4,7,10-tetraazacyclododecane (CNBQ). Three separate methods, $\mathrm{pH}$-metric, UV-metric, and Reverse Phase-High Performance Liquid Chromatography (RP-HPLC), were employed to determine the pKa values between $2.0-12.0 \mathrm{pH}$ range. The acetate and phosphate buffers, in addition to methanol and acetonitrile as co-solvents and potassium chloride to maintain the ionic strength, were used as appropriate. In UV-metric method, the drug substance is dissolved in aqueous media eliminating any interference of a co-solvent for measuring the $\mathrm{pKa}$. Consequently, the pKa values obtained by the UV-metric method are considered accurate, as opposed to potentiometric and RP-HPLC methods that require the use of co-solvents. Thus, through the utilization of UV-metric method three pKa values, 5.9, 6.6, and 8.7, were obtained for CNBQ. These studies would be useful to determine the pKa values of the related drug leads under development.
\end{abstract}

Keywords: pKa; pH-metric; UV-metric; RP-HPLC methods; Drug lead; Drug development

\section{Introduction}

The acid dissociation constant (pKa) is the $\mathrm{pH}$ at which concentrations of ionized and unionized forms of drugs are equal. It is an essential parameter in drug discovery, particularly in physiological systems where ionization state will affect the rate at which the compound is able to diffuse across membranes including blood-brain barrier [1]. Unless the drug is given intravenously, the drug must pass through several different semipermeable membranes before it reaches the systemic circulation and later in the site of action. The semipermeable cell membranes, due to their inherent hydrophobicity, selectively inhibit the passage of drug molecules. The simplest route for a drug to enter the systemic circulation is by passive diffusion through the semipermeable cell membranes from an area of high concentration to an area of low concentration. This requires the ingenuity from drug developers to design drug substances that will overcome these obstacles and allow the proper passage through the semipermeable membranes.

Most drugs are weak organic acids or bases existing in ionized and unionized forms in aqueous solutions. The unionized forms of drugs are usually more lipophilic and thus readily diffuse across cell membranes. It is in this instance that $\mathrm{pKa}$ and bioavailability correlate. When the $\mathrm{pH}$ is lower than the $\mathrm{pKa}$, the unionized form of a weak acid predominates, and vice versa for a weak base [2]. Proceeding with this concept, different $\mathrm{pH}$ in the body allow for the alteration of solubility, dissociation, and coincident absorption of the drug substance [3] by shifting the concentration of the unionized and ionized forms of drug substances, therefore, validating the importance of determining the pKa of a drug.

The antimalarial drug lead cyclen bisquinoline (CNBQ; Figure 1) demonstrated a potent in vitro anti-malarial activity against chloroquine-sensitive and chloroquine-resistant as well as mefloquineresistant strains of Plasmodium falciparum. The compound was also found to be a potent antimalarial agent in vivo [4]. Moreover, the drug lead was found to be metabolically stable in vitro in the presence of HLM and cDNA expressing CYP2C8 enzymes [5]. As malaria is caused by invasion of malaria parasites in the blood, it is important that the drug substance intended to treat it is able to enter the systemic circulation. Therefore, evaluation of $\mathrm{pKa}$ values is imperative in the early stage of drug discovery and development, because, as previously mentioned, $\mathrm{pKa}$ values of the drug substance and its bioavailability are correlated. There are several different methods that can be employed to determine the $\mathrm{pKa}$ values of the drugs, such as: $\mathrm{pH}$-metric, $\mathrm{UV}$-metric, NMR, solubility, capillary electrophoresis, HPLC, conductometry, voltammetry, calorimetry, fluorimetry, polarimetry, kinetic, and computational methods [6]. It is challenging to find a single method that will not only measure any and all pKa values, but also be reliable in its findings in just one attempt due to the vast amount of variability in drug structures. This paper will focus on the following three methods: $\mathrm{pH}$-metric, UV-metric, and the RP-HPLC methods. Many of other methods previously mentioned require a substantial amount of instrumentation, test material and/or time, making them unsuitable or not feasible for implementation in this research lab (Figure 1).

\section{Materials and Methods}

\section{Materials}

CNBQ (Figure 1) was synthesized in our laboratory and the purity checked by HPLC [7]. Chloroquine diphosphate was purchased from Pfaltz \& Bauer. The solvents and reagents used were as follows: acetonitrile, methanol, sodium hydroxide, potassium hydroxide, hydrochloric acid, potassium chloride, ammonium acetate, and sodium phosphate dibasic anhydrous. Each solvent and reagent used was HPLC and analytical grade and was purchased from Fisher Scientific. Deionized water used to prepare the solutions and mobile phase was further purified by filtration and degassing.

*Corresponding author: Faruk Khan MO, SCRiPS, College of Pharmacy, Southwestern Oklahoma State University, 100 Campus Drive, Weatherford, OK 73096, USA, Tel: 580-774-3064; E-mail: faruk.khan@swosu.edu

Received December 15, 2014; Accepted December 26, 2014; Published December 30, 2014

Citation: Hossain MF, Obi C, Shrestha A, Khan MOF (2014) UV-Metric, pH-Metric and RP-HPLC Methods to Evaluate the Multiple pKa Values of a Polyprotic Basic Novel Antimalarial Drug Lead, Cyclen Bisquinoline. Mod Chem appl 2: 145. doi: 10.4172/2329-6798.1000145

Copyright: (c) 2014 Hossain MF, et al. This is an open-access article distributed under the terms of the Creative Commons Attribution License, which permits unrestricted use, distribution, and reproduction in any medium, provided the original author and source are credited. 


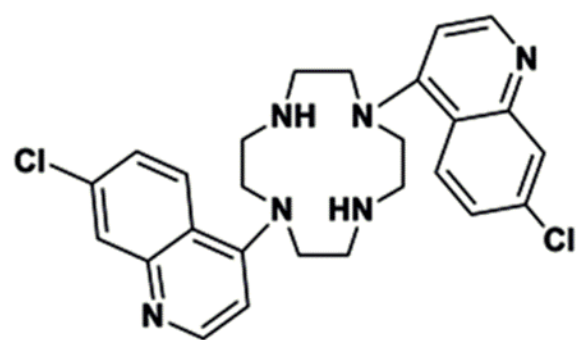

Figure 1: 4,10-bis (7-chloroquinoline)-1,4,7,10-tetraazacyclododecane (Cyclen isquinoline, $\mathrm{CNBQ}$

\section{Methods}

pH-metric method (Potentiometric titration): The $\mathrm{pH}$-metric titration was performed using two different instruments, highly sensitive fully automated Sirius T3 in Sirius laboratory and semiautomated Metrohm in our laboratory. In Sirius laboratory, $0.01 \mathrm{M}$ phosphate buffer was used to prepare the drug solution, and $\mathrm{pH}$ of the buffer were accurately adjusted from $\mathrm{pH} 2.0$ to 12.0 with 0.2 interval using $0.5 \mathrm{M} \mathrm{HCl}$ and $0.5 \mathrm{M} \mathrm{KOH}$ titrants as appropriate. The $\mathrm{pH}$ electrode of the potentiometer was calibrated using standard buffers at pHs of 4.0, 7.0 and 10.0. 0.1 M hydrochloric acid was used to prepare the drug solution, and $\mathrm{pHs}$ of the buffer was adjusted from $\mathrm{pH} 2.0$ to 12.0 using $0.1 \mathrm{M} \mathrm{NaOH} / 0.1 \mathrm{M} \mathrm{KOH}$ titrants as appropriate. In both cases, the ionic strength of the solution was maintained using $0.15 \mathrm{M}$ potassium chloride solution and methanol was used as a co-solvent. The solution temperature was set at $25^{\circ} \mathrm{C}$ and nitrogen purging was performed to displace the dissolved gases from titrating solutions in both experiments.

In the $\mathrm{pH}$-metric titration, a known volume of reagent is added in a step wise mode to the analyte. The change in the measure of potential is determined by the use of two electrodes, an indicator and a reference electrode [8]. The changes in potential vs. $\mathrm{pH}$ are graphed; subsequently producing a sigmoidal curve allowing the determination of the $\mathrm{pKa}$ of the compound. The $\mathrm{pKa}$ value is the $\mathrm{pH}$ at half-neutralization point, which represents the center point on the ascending portion of the sigmoidal curve, and was integrated by automated software.

UV-metric method (Spectrophotometric determination): Spectrophotometric determination of $\mathrm{pKa}$ was performed using two different instruments, highly sensitive fully automated Sirius T3 in Sirius laboratory and semi-automated NanoDrop 2000c Spectrophotometer in our laboratory. With the implementation of Sirius T3 instrument, the sample was subsequently titrated in a UV-metric triple titration from $\mathrm{pH} 2.0-12.0$ at concentrations of $11-16 \mu \mathrm{M}$ under aqueous conditions (0.01 M Phosphate Buffer). The ionic strength of the solution was maintained using $0.15 \mathrm{M}$ potassium chloride solution. The buffer allowed for controlled $\mathrm{pH}$ as it was adjusted from $\mathrm{pH} 2.0$ to 12.0 using $0.5 \mathrm{M} \mathrm{HCl}$ and $0.5 \mathrm{M} \mathrm{KOH}$ titrants. The $\mathrm{pKa}$ values were determined using Dip-Probe Absorption Spectroscopy (D-PAS) technique. In the D-PAS technique, a fiber optics dip-probe, a UV-light source (Deuterium Light), and a photodiode array detector were used in conjunction with a titrator to capture the spectral changes which arise during the course of titration. Software set up all experimental data in an absorbance matrix based on Beer's law (Absorbance $=$ Concentration $\times$ Extinction Coefficient), and Target Factor Analysis (TFA, at the rate of change at which the compound's UV absorbance was the strongest) detected the corresponding $\mathrm{pKa}$ values from the absorbance matrix [9].

The second experiment utilized the NanoDrop Spectrophotometer instrument and $1 \mathrm{~cm}^{2} \mathrm{UV}$-cell. UV spectra were taken throughout the course of titration with an approximate interval of $0.2 \mathrm{pH}$ unit, between $\mathrm{pH} 3.0$ - 11.0 at concentrations of $0.05 \mathrm{mM}$ under aqueous conditions (0.01M Phosphate Buffer). The ionic strength of the solution was maintained using $0.15 \mathrm{M}$ potassium chloride solution. The $\mathrm{pH}$ of the buffer was adjusted by addition of $0.5 \mathrm{M} \mathrm{HCl}$ and $0.5 \mathrm{M} \mathrm{KOH}$ solution using ACCUMET $\mathrm{pH}$ meter. The corresponding $\mathrm{pKa}$ values were determined by visual evaluation based on changes in the compound's UV spectra pattern with respect to $\mathrm{pH}$. The $\mathrm{pKa}$ value corresponds to the $\mathrm{pH}$ at which the rate of change of the UV spectra of the compound is the strongest.

RP-HPLC method: To determine the pKa using RP-HPLC, 0.084 $\mathrm{mg} / \mathrm{ml}$ solution of CNBQ as a test sample, and $0.032 \mathrm{mg} / \mathrm{ml}$ solution of chloroquine diphosphate as a reference sample were prepared in a 50:50 solution of acetonitrile and water. The solutions were then injected using the following chromatographic conditions to determine the retention time in different $\mathrm{pH}$ values of the mobile phase. The $\mathrm{pKa}$ values were determined from the first derivative curve of the retention time vs. $\mathrm{pH}$ values.

Chromatographic separation of CNBQ and chloroquine were successfully achieved on a Waters X-Bridge C-18 column $(4.6 \mathrm{~mm} \times$ $250 \mathrm{~mm}, 5.0 \mu \mathrm{m}$ particle sizes, part no. 186003117) purchased from Waters Corporation in an isocratic separation mode with a mobile phase consisting of $50 \%$ of acetonitrile and $50 \%$ of $0.002 \mathrm{M}$ ammonium acetate. The $\mathrm{pH}$ of the mobile phase was set within the range of 7.012.0 with an interval of 0.2 with $0.1 \mathrm{~N} \mathrm{HCl}$ and $0.1 \mathrm{~N} \mathrm{NaOH}$. The flow rate was maintained at $1.0 \mathrm{ml} / \mathrm{min}$, the column oven temperature was maintained at $25^{\circ} \mathrm{C}$, the injection volume was set at $1 \mu \mathrm{L}$, and the effluent was monitored at $254 \mathrm{~nm}$.

\section{Results and Discussion}

As mentioned previously, the pKa values of CNBQ were determined using the following three methods: $\mathrm{pH}$-metric, UV-metric and RP-HPLC. Considering the variability in the results obtained from the three aforementioned methods, three pKa values: 5.9, 6.6, and 8.7 were estimated for the compound. Table 1 represents the $\mathrm{pKa}$ values observed and calculated from these methods.

The potentiometric determination of $\mathrm{pKa}$ is a relatively simple, which can be used for any ionizable compound that does not require presence of chromophore groups for $\mathrm{pKa}$ determination [10]. pKa determination by this method is the most economical method, in regard to the short duration of experiment and the ease of reproducibility if carried out correctly. Limitations of this method has been the need for a higher amount of drug substance in order to achieve an accurate result and necessity for the use of co-solvents for poorly water soluble basic compound to prevent precipitation of the drug at higher $\mathrm{pH}$ values. $\mathrm{CNBQ}$, being insoluble at higher $\mathrm{pH}$, requires extrapolation using methanol as a co-solvent. Use of a co-solvent impacts the $\mathrm{pKa}$ values [11], resulting in a higher level of variability than normally observed.

Table 2 represents the data obtained by $\mathrm{pH}$-metric method. $\mathrm{pKa}$ values of CNBQ was determined using Yasuda-Shedlovsky standard extrapolation method [12] as shown in Figure 2, in which X-axis plots the inverse of the dielectric constant of the water-solvent mixture at the experimental percentage of solvent and $\mathrm{Y}$-axis plots the psKa $+\log \left[\mathrm{H}_{2} \mathrm{O}\right]$; where, psKa is cosolvent dissociation constants, and $\left[\mathrm{H}_{2} \mathrm{O}\right]$ is the molar water concentration of the given solvent mixture. The extrapolated value represents the $\mathrm{pKa}$ value at $100 \%$ water concentration ( $0 \%$ co-solvent). Sirus T3 software could conveniently calculate the extrapolated $\mathrm{pKa}$ values in water using this method. The pKa values of CNBQ were determined by this method to be 5.8, 6.4 and 


\begin{tabular}{|c|c|c|c|}
\hline $\mathbf{p K a}$ Values & $\mathbf{p K}_{\mathrm{a} 1}$ & $\mathbf{p K}_{\mathrm{a} 2}$ & $\mathbf{p K}_{\mathrm{a} 3}$ \\
\hline UV-metric method (Automated) & $5.87 \pm 0.01$ & $6.60 \pm 0.01$ & $8.73 \pm 0.01$ \\
\hline pH-metric method (Automated) & $5.80 \pm 0.01$ & $6.35 \pm 0.11$ & $8.43 \pm 0.03$ \\
\hline UV-metric method (Manual) & 5.99 & 6.81 & 8.67 \\
\hline RP-HPLC method & --- & --- & 8.8 \\
\hline
\end{tabular}

Table 1: pKa values of CNBQ by different methods

\begin{tabular}{|l|c|c|c|c|c|c|}
\hline pKa Values & \%Methanol & $\begin{array}{c}\text { Dielectric } \\
\text { Constant }\end{array}$ & $\left.\mathbf{[ H}_{\mathbf{2}} \mathbf{O}\right]$ & psK $_{\mathrm{a} 1}$ & psK $_{\mathrm{a} 2}$ & psK $_{\mathrm{a} 3}$ \\
\hline Experiment 1 & 29.2 & 65.9 & 36.4 & 5.4 & 6.0 & 8.3 \\
\hline Experiment 2 & 38.9 & 61.5 & 30.6 & 5.2 & 5.9 & 8.2 \\
\hline Experiment 3 & 49.5 & 56.6 & 24.7 & 4.9 & 5.6 & 8.2 \\
\hline
\end{tabular}

Table 2: $\mathrm{pKa}$ values of $\mathrm{CNBQ}$ by $\mathrm{pH}$-metric method using Sirius $\mathrm{T} 3$

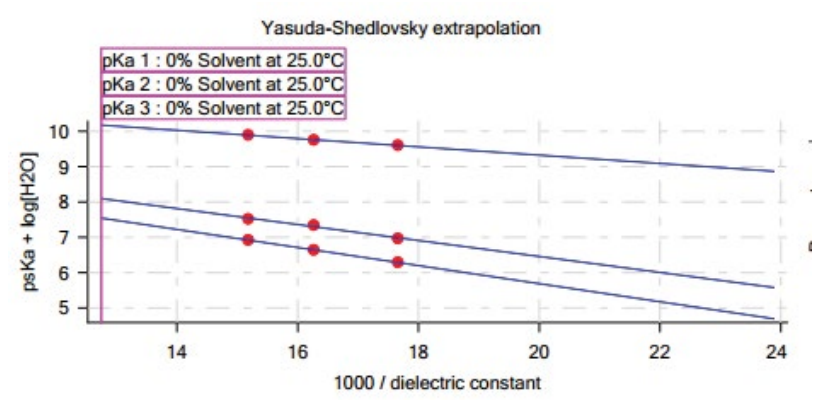

Figure 2: Yasuda-Shedlovsky standard extrapolation in pH-metric method by Sirius T3

8.4 with a $\mathrm{R}^{2}$ value of 0.99 . Another attempt was made to determine the pKa value of CNBQ using a Metrohm potentiometer in our laboratory. In this experiment, methanol and acetonitrile were used as co-solvents. Although the experiment was performed in a controlled setting, it did not produce any reproducible $\mathrm{pKa}$ value due to poor solubility of the basic compound causing precipitation of the samples in the solution as $\mathrm{pH}$ is increased, and lack of sensitivity of the instrument. In general, a higher quantity of the sample is required to determine the pKa using an ordinary potentiometer that causes precipitation of the sample and thus inaccurate results. However, due to recent advances in technology (Sirius T3, as discussed above), pKa values can now be determined in a more economical approach using smaller amounts of sample, which is very important in the early stage of drug development (Figure 2 and Table 2) [13].

RP-HPLC method was developed to overcome the challenges posed by the properties of current drugs with low solubility. This method is attractive due to its simplicity and its ability to use a variety of isocratic HPLC systems with the use of less drug material [14].pKa is determined by analyzing the change in retention time of an analyte vs. the $\mathrm{pH}$ of the mobile phase of the respective retention time. Utilization of RPHPLC method has proven to be successful in determining pKa values that resemble literature $\mathrm{pKa}$ values $[15,16]$. The fundamental principle of this method is the variation of retention time based on the $\mathrm{pH}$ of the mobile phase. However, obtaining a sharp peak shape and accurate retention time at each point using an isocratic mobile phase in the $\mathrm{pH}$ range of 2.0 to 12.0 is quite difficult when dealing with polyprotic basic compound having multiple $\mathrm{pKa}$ values. Improvement of peak shape can be accomplished by addition of an organic modifier and an ion pairing reagent to the mobile phase, but addition of these reagents will result in inaccurate $\mathrm{pKa}$ values [14]. CNBQ is a polyprotic basic drug and shows a broader peak shape in the lower $\mathrm{pH}$ ranges, which are not reproducible. It was revealed in this experiment that good peak shape for CNBQ can be obtained within the $\mathrm{pH}$ range of 7.0 to 12.0 as shown in Figure 3. Thus, the $\mathrm{pKa}$ determination was performed in the $\mathrm{pH}$ range of 7.0 to 12.0 , using acetonitrile as an organic phase and acetate buffer as the aqueous phase. Because of the structural similarities between chloroquine and CNBQ, chloroquine was used as a reference throughout the entire experiment. The $\mathrm{pKa}$ values for both compounds were determined from the first derivative curve of the retention time vs. $\mathrm{pH}$ values (Table 3 and Figure 4). The pKa values for chloroquine obtained by RP-HPLC method were 8.4 and 10.2 (compared to the literature values of 8.37 and 10.49 , respectively) [12]. In regards to CNBQ, RP-HPLC method only produced one pKa value of 8.8 , which proves the difficultly in determining multiple $p K a$ values using RPHPLC method beyond the range of 7-12 as mentioned before figures 3 and 4 .

The UV-metric method measures absorption, emission, or scattering of electromagnetic radiation with respect to changes in $\mathrm{pH}$, leading to changes in the UV spectra of the molecule [17]. These changes in the multi-wavelength UV spectra can be observed, if the disposition of electrons in chromophore containing conjugated double bonds, carbonyl groups, and other UV absorbing groups change with the molecule's ionization state. There are several methods to calculate the pKa values from UV absorbance data vs. $\mathrm{pH}[13,17-19]$. There are two disadvantages of the UV-metric method. First, if the compound is too basic it may precipitate out; however, this can be overcome if the experiment is performed in the presence of a co-solvent, but would require extrapolation to obtain the final results. Second, if the compound possesses no $\mathrm{pH}$-active chromophore, then the UV-metric method cannot be applied making this the major disadvantage. If the sample has a UV response and exhibits no/less precipitation, then it is possible to conduct the experiment under aqueous conditions using only small amount of the compound [13]. This results in pKa values which are considered more accurate, because there is no interference of a co-solvent and no extrapolation is needed to obtain the final results. In this experiment using automated software of Sirius T3 instrument, TFA detected the corresponding pKa values at which the rate of change in the compound's UV absorbance is the strongest. CNBQ exists in four different species; $\mathrm{BH} 3, \mathrm{BH} 2, \mathrm{BH}$, and $\mathrm{B}$ at depending on the $\mathrm{pH}$ of the medium. Figure 5 represents the spectra for four different species of CNBQ obtained by Sirius T3 instrument. Utilizing this method, three pKa values of 5.87, 6.60, and 8.73, were observed from the spectroscopic data. Table 4 represents the data obtained from automated UV-metric method.

Figure 6 represents the spectra of different species of CNBQ obtained from NanoDrop 2000c Spectrophotometer at different pHs. In this figure, $\mathrm{pKa}_{1} \mathrm{pKa}_{2}$ and $\mathrm{pKa}_{3}$ represent the strongest changes of spectrum from one species to another species. The corresponding $\mathrm{pKa}$ values of $5.95,6.83$, and 8.67 , were determined by visual evaluation based on changes in the compound's UV spectra pattern with respect to $\mathrm{pH}$ (Table 5 and Figure 6). When the pKa values obtained by this manual UV-metric method, conducted in our lab, plotted against those obtained by fully automated Sirius T3 instrument, it exhibited a straight line with $\mathrm{R}^{2}$ value of 0.993 (Figure 7), signifying the accuracy and validity of the results. Figure 8 represents the four different spectra for the species of CNBQ obtained by NanoDrop 2000c Spectrophotometer (Table 5).

Figure 9 represents the distribution of species of CNBQ in different $\mathrm{pH}$ conditions by Henderson-Hasselbalch equation. From the species distribution it is clearly shown that the lone pairs of electrons of nitrogen atoms in the aliphatic and aromatic ring of CNBQ will fully accept three protons (BH3 Species) at lower $\mathrm{pH}$ (approximately $\mathrm{pH}$ 4.0). CNBQ will be partially ionized at an approximate $\mathrm{pH}$ of 6.0 ( $\mathrm{BH} 2$ 
Citation: Hossain MF, Obi C, Shrestha A, Khan MOF (2014) UV-Metric, pH-Metric and RP-HPLC Methods to Evaluate the Multiple pKa Values of a Polyprotic Basic Novel Antimalarial Drug Lead, Cyclen Bisquinoline. Mod Chem appl 2: 145. doi:10.4172/2329-6798.1000145

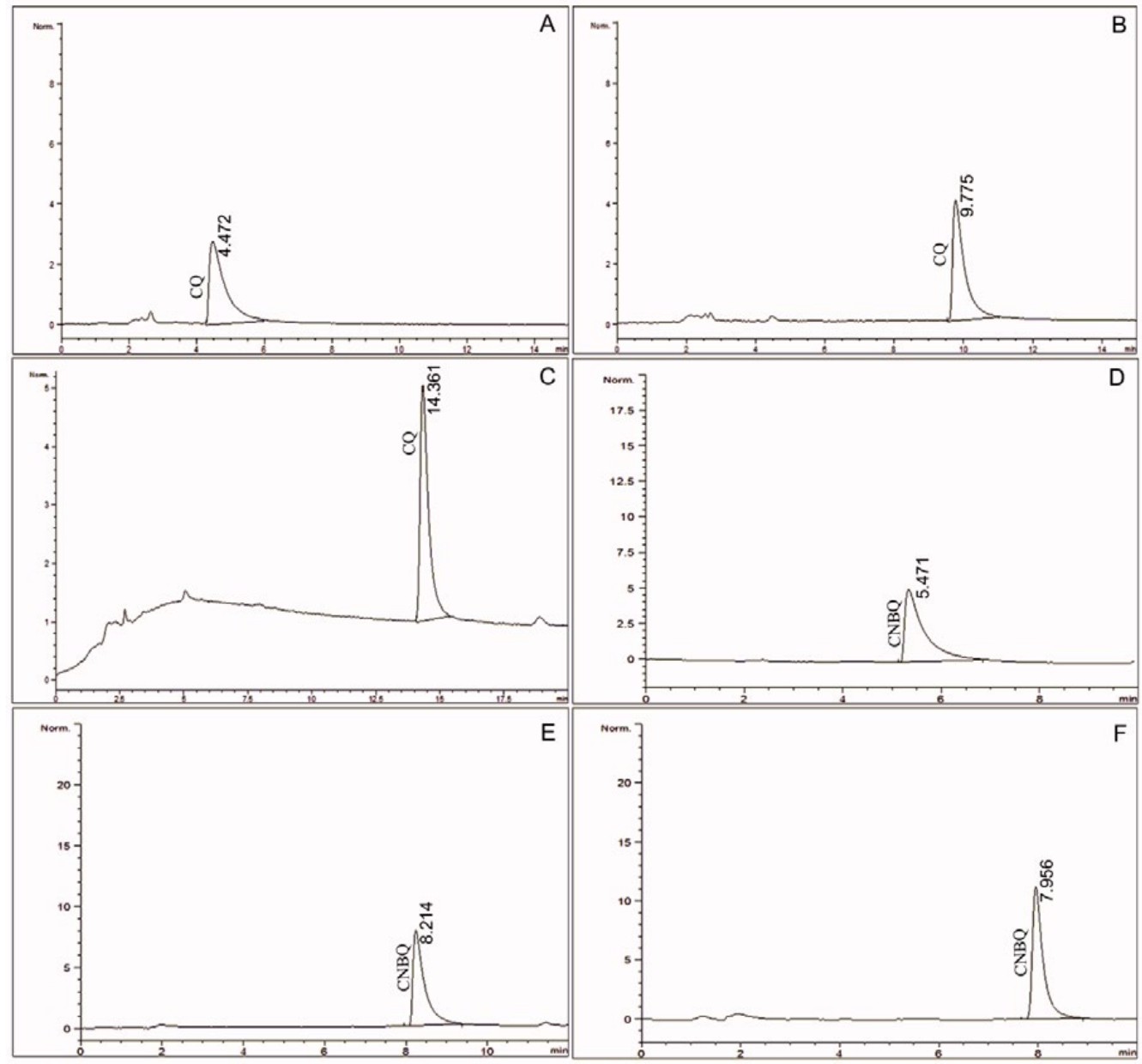

Figure 3: HPLC Chromatograms; $\mathrm{A}: \mathrm{CQ}$ at $\mathrm{pH}$ 7.3, $\mathrm{B}: \mathrm{CQ}$ at $\mathrm{pH}$ 9.5, C: $\mathrm{CQ}$ at $\mathrm{pH} 11.1, \mathrm{D}: \mathrm{CNBQ}$ at $\mathrm{pH} 7.5, \mathrm{E}: \mathrm{CNBQ}$ at $\mathrm{pH}$ 9.4, F: CNBQ at $\mathrm{pH} 11.1$

\begin{tabular}{|c|c|c|c|c|c|}
\hline \multicolumn{3}{|c|}{ Chloroquine } & \multicolumn{3}{|c|}{ CNBQ } \\
\hline RT & $\mathrm{pH}$ & $\Delta R T / \Delta p H$ & RT & $\mathrm{pH}$ & $\Delta R T / \Delta p H$ \\
\hline 4.472 & 7.31 & --- & 5.338 & 6.97 & --- \\
\hline 4.460 & 7.50 & 0.179 & 5.471 & 7.49 & 0.256 \\
\hline 4.528 & 7.88 & 0.311 & 5.647 & 7.64 & 1.173 \\
\hline 4.584 & 8.06 & 0.926 & 6.000 & 7.88 & 1.471 \\
\hline 4.797 & 8.29 & 1.931 & 6.341 & 8.13 & 1.364 \\
\hline 5.048 & 8.42 & 3.700 & 6.774 & 8.41 & 1.546 \\
\hline 5.455 & 8.53 & 3.231 & 7.094 & 8.60 & 1.684 \\
\hline 5.875 & 8.66 & 3.604 & 7.414 & 8.75 & 2.133 \\
\hline 7.533 & 9.12 & 5.468 & 7.734 & 8.84 & 3.556 \\
\hline 9.775 & 9.53 & 5.715 & 8.023 & 9.14 & 0.963 \\
\hline 12.518 & 10.01 & 3.894 & 8.214 & 9.42 & 0.682 \\
\hline 13.180 & 10.18 & 6.417 & 8.278 & 9.64 & 0.291 \\
\hline 13.950 & 10.30 & 1.477 & 8.286 & 9.74 & 0.080 \\
\hline 14.334 & 10.56 & 0.055 & 8.284 & 9.97 & -0.009 \\
\hline 14.361 & 11.05 & -0.178 & 7.956 & 11.07 & -0.298 \\
\hline
\end{tabular}

Table 3: Retention time (RT) and $\mathrm{pH}$ of the mobile phase

Species) and at an approximate $\mathrm{pH}$ of 8.0 (BH Species), accepting two and one proton(s), respectively. CNBQ will be completely unionized (B Species) at higher $\mathrm{pH}$ (approximately $\mathrm{pH} 10.0)$.

To validate the $\mathrm{pKa}$ values obtained using in-house RP-HPLC method, chloroquine was used as a reference. The $\mathrm{pH}$-metric method

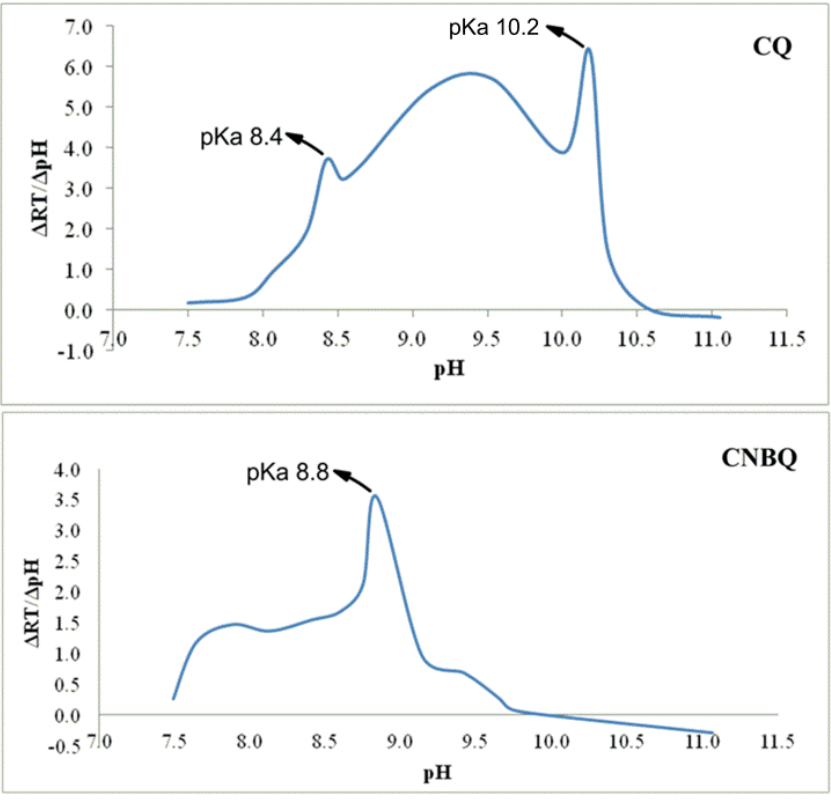

Figure 4: First derivative curves for pKa determination; Top, chloroquine, bottom, CNBQ 

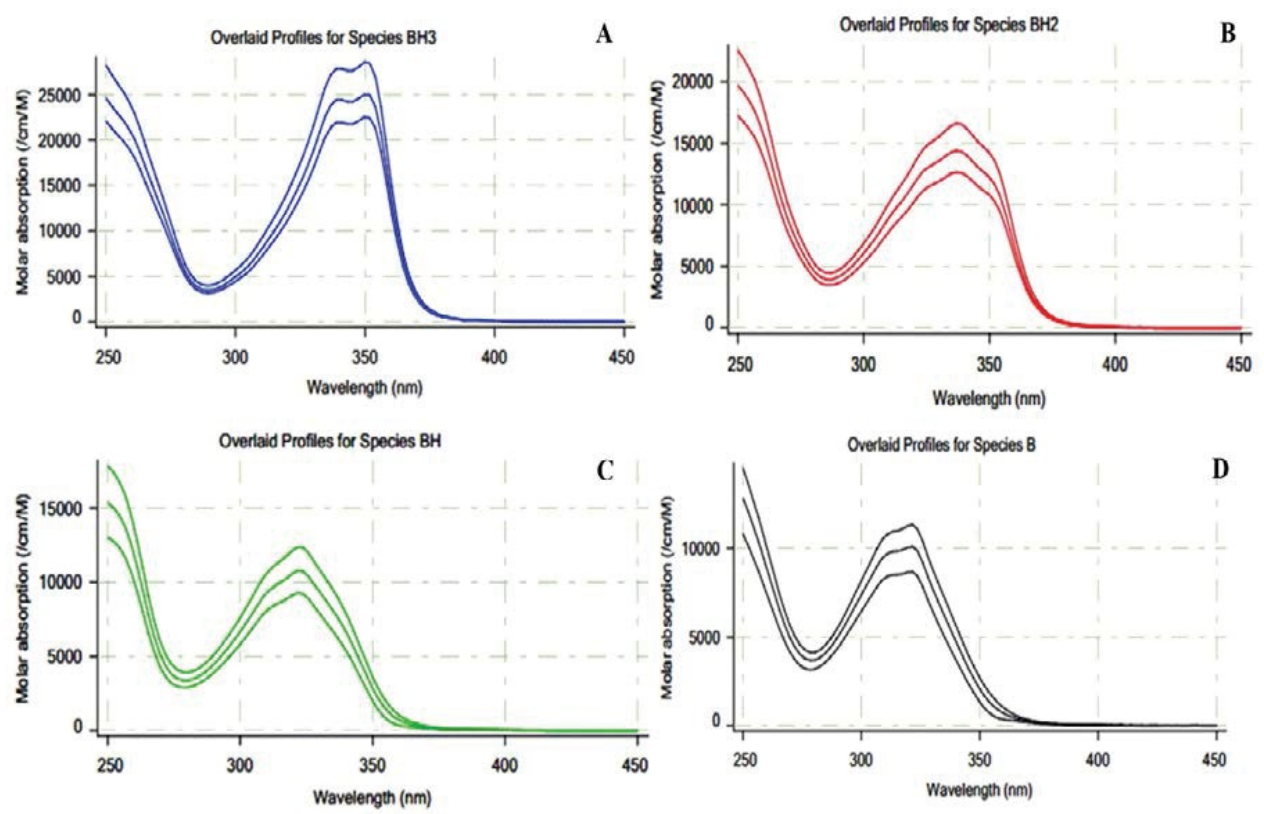

Figure 5: Spectra of different Species of $\mathrm{CNBQ}$ obtained from Sirius T3; $\mathrm{A}: \mathrm{BH}$, B: BH2, C: $\mathrm{BH}$ and D: B

\begin{tabular}{|c|c|c|c|}
\hline pKa Values & $\mathbf{p K}_{\mathrm{a} 1}$ & $\mathbf{p K}_{\mathrm{a} 2}$ & $\mathbf{p K}_{\mathrm{a} 3}$ \\
\hline Experiment 1 & 5.87 & 6.59 & 8.73 \\
\hline Experiment 2 & 5.89 & 6.61 & 8.73 \\
\hline Experiment 3 & 5.86 & 6.60 & 8.73 \\
\hline Average & 5.87 & 6.60 & 8.73 \\
\hline Std Dev & 0.012 & 0.007 & 0.004 \\
\hline
\end{tabular}

Table 4: pKa values of CNBQ by UV-metric method using Sirius T3

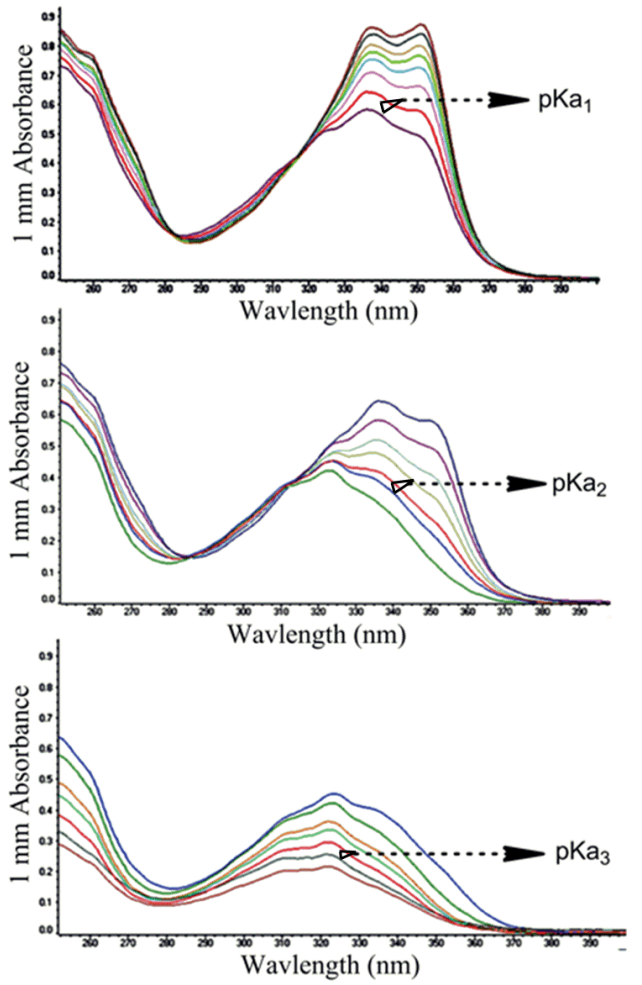

Figure 6: Spectra of different Species of $C N B Q$ obtained from NanoDrop Spectrophotometer at changing $\mathrm{pHs}$

\begin{tabular}{|c|c|c|c|}
\hline $\mathbf{p K a}$ Values & $\mathbf{p K}_{\mathrm{a} 1}$ & $\mathbf{p K}_{\mathrm{a} 2}$ & $\mathbf{p K}_{\mathrm{a} 3}$ \\
\hline \multirow{2}{*}{$\mathrm{pH}$ of the two consecutive Spectra } & 5.85 & 6.74 & 8.56 \\
\cline { 2 - 4 } & 6.05 & 6.91 & 8.77 \\
\hline Average & 5.95 & 6.83 & 8.67 \\
\hline
\end{tabular}

Table 5: pKa values of CNBQ by UV-metric method using NanoDrop spectrophotometer

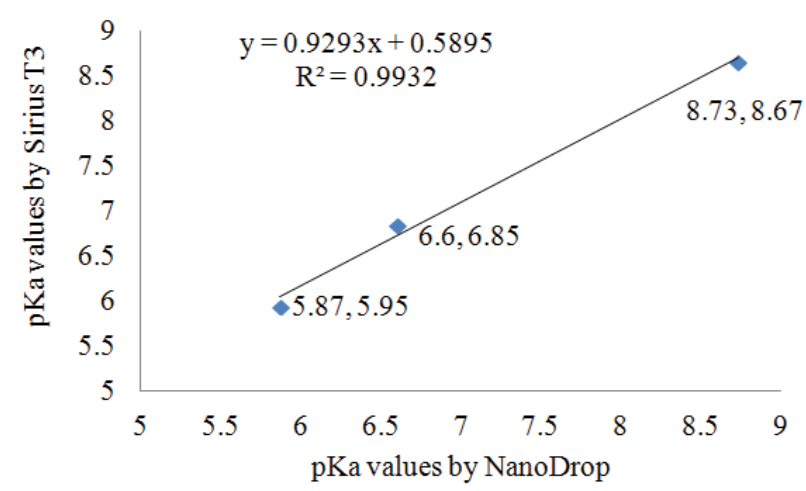

Figure 7: A plot of the pKa values obtained using NanoDrop spectrophotometer against those measured by Sirius T3

and the D-PAS technique for UV-metric method has already been validated using several compounds by Sirius Analytical Ltd. $[9,13]$. Therefore, UV-metric method using visual inspection was validated by comparing the results obtained at both laboratories (Figure 7). The pKa values obtained from both methods were within \pm 0.2 with a $\mathrm{R}^{2}$ value of 0.99 . These minor variations are due to changes in instruments, differences in analytical reagents, and different analyst. Since the compound has excellent UV signal and the analysis was performed under aqueous conditions, it did not require an extrapolation. Hence, UV-metric method stands out as an effective method for the determination of multiple $\mathrm{pKa}$ values of a poorly water-soluble, polyprotic basic compound having good UV signal. 

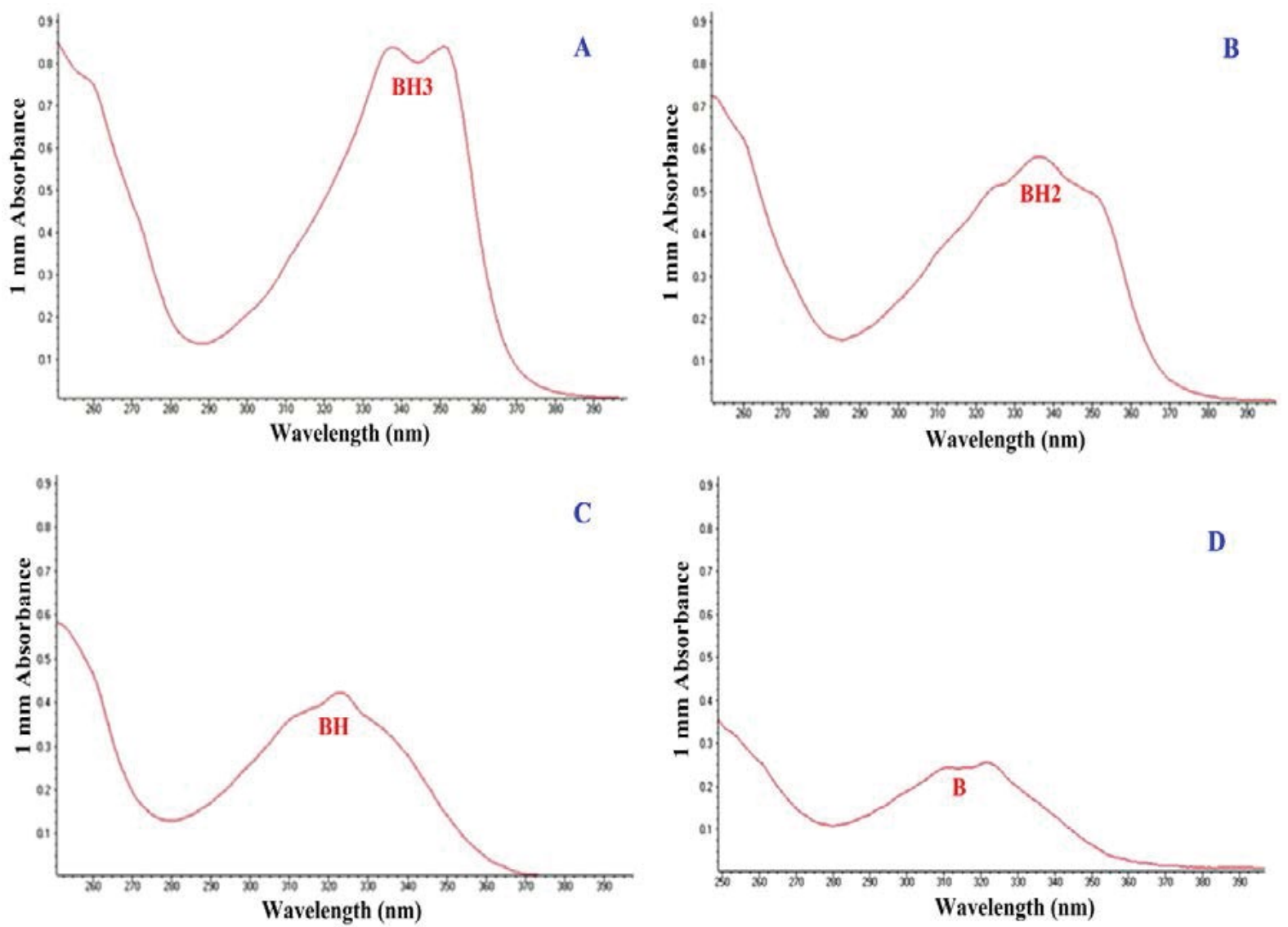

Figure 8: Spectra of different Species of CNBQ obtained from NanoDrop Spectrophotometer; A: BH3, B: BH2, C: BH and D: B
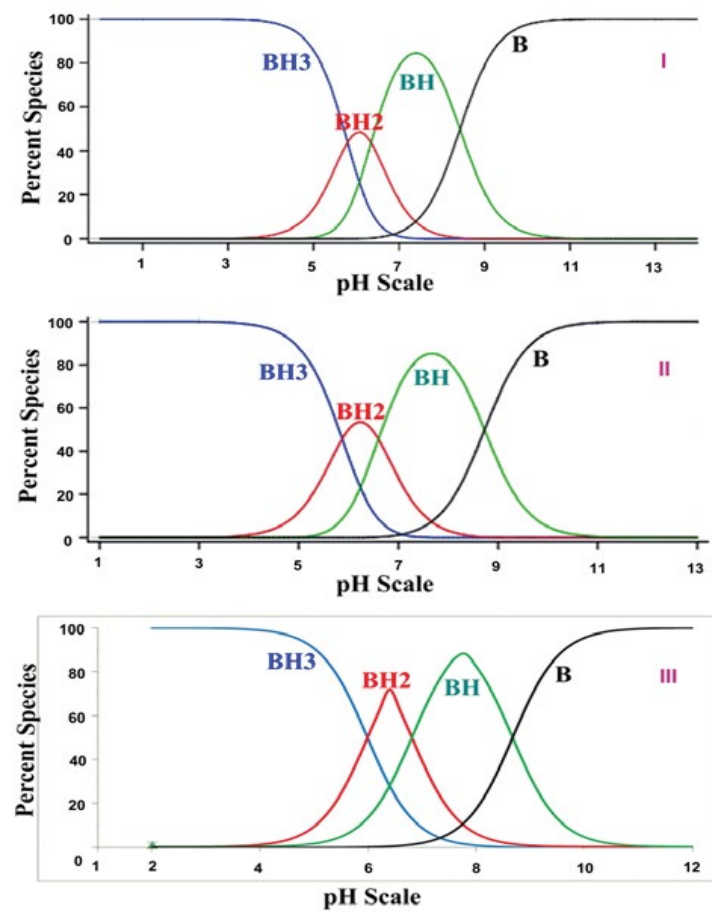

Figure 9: Distribution of species of CNBQ; I: $\mathrm{pH}$-metric method (Sirius T3), II: UV-metric method (Sirius T3), III: UV-metric method (NanoDrop)

\section{Conclusion}

From the pKa values obtained, it is apparent that CNBQ is a polyprotic basic drug in which the lone pairs of electrons on nitrogen atoms in aliphatic and aromatic ring will accept three protons in different physiological $\mathrm{pH}$ conditions. It is imperative that the method chosen is the most accurate method in determining these $\mathrm{pKa}$ values. Considering all the methods discussed, UV-metric method is the most suitable in determining the pKa values for the drug lead CNBQ due to its basicity, inherent insolubility, and multiple pKa values. The RPHPLC method possesses limitations, in regards to the $\mathrm{pH}$ range $(\mathrm{pH} 7.0$ to 12.0 as observed in our experiment), of the mobile phase, making it incapable of determining $\mathrm{pKa}$ values that fall outside the range. Use of co-solvents complicates results obtained from both RP-HPLC and $\mathrm{pH}$ metric methods, as the co-solvent will produce a pKa value that is not accurate compared to the actual value. Furthermore, results obtained from $\mathrm{pH}$-metric and RP-HPLC methods require extrapolation making them variable and less accurate than those obtained from the UVmetric method, which does not require co-solvents or extrapolation. In conclusion, RP-HPLC and $\mathrm{pH}$-metric methods are not suitable methods for determining $\mathrm{pKa}$ values of drug leads similar to CNBQ. However, UV-metric method can be conveniently and accurately utilized to determine the pKa values of drug leads of this class in our laboratory.

\section{Acknowledgment}

This project was supported by the National Institute of General Medical Sciences of the National Institutes of Health through Grant Number 8P20GM103447. 
Citation: Hossain MF, Obi C, Shrestha A, Khan MOF (2014) UV-Metric, pH-Metric and RP-HPLC Methods to Evaluate the Multiple pKa Values of a Polyprotic Basic Novel Antimalarial Drug Lead, Cyclen Bisquinoline. Mod Chem appl 2: 145. doi:10.4172/2329-6798.1000145

Page 7 of 7

\section{References}

1. Manallack DT (2007) The pKa distribution of drugs: Application to drug discovery. Perspect Medicin Chem 1: 25-38.

2. http://www.merckmanuals.com/

3. Chen XQ, Antman MD, Gesenberg C, Gudmundsson OS (2006) Discovery pharmaceutics-challenges and opportunities. AAPS J 8: E402-E408.

4. Khan MOF, Levi MS, Tekwani BL, Khan SI, Kimura E, et al. (2009) Synthesis and antimalarial activities of cyclen 4-aminoquinoline analogs. Antimicrob Agents Chemother 53: 1320-1324.

5. Rudraraju AV, Hossain MF, Shrestha A, Amoyaw PNA, Tekwani BL, et al. (2014) In vitro metabolic stability study of new cyclen based antimalarial drug leads using RP-HPLC and LC-MS/MS. Mod Chem appl 2: 129.

6. Reijenga J, Van hoof A (2013) Development of methods for the determination of pKa values. Anal Chem Insights 8: 53-71.

7. Amoyaw PNA, Pham K, Cain AN, McClain JM, Khan MOF, et al. (2014) Synthesis of novel tetraazamacrocyclic bisquinoline derivatives as potential antimalarial agents. Curr Org Synth 11: 916-921.

8. Qiang Z, Adams C (2004) Potentiometric determination of acid dissociation constants $\left(\mathrm{pK}_{\mathrm{a}}\right)$ for human and veterinary antibiotics. Water Res 38: 2874-2890.

9. Tam KY, Takács-Novak K (2001) Multi-wavelength spectrophotometric determination of acid dissociation constants: a validation study. Analytica Chimica Acta 434: 157-167.
10. Demiralay EC, Yılmaz H (2012) Potentiometric pK determination of piroxicam and tenoxicam in acetonitrile-water binary mixtures. SDU J Sci 7: 34-44.

11. Kamble AD, Barhate, VD, Salunke MH, Narasimham L (2011) Automated potentiometric titration method for determination of pKa values: An application to hydroxy chloroquine sulphate. J Pharm Res 4: 3794-3797.

12. http://www.sirius-analytical.com

13. Box KJ, Comer JEA (2008) Using measured pKa, $\log P$ and solubility to investigate supersaturation and predict BCS Class. Curr Drug Metab 9: 869878

14. Manderscheid M, Eichinger T (2003) Determination of pKa values by liquid chromatography. J Chromatogr Sci 41: 323-326.

15. Wiczling P, Markuszewski MJ, Kaliszan R (2004) Determination of pKa by pH gradient reversed-phase HPLC. Anal Chem 76: 3069-3077.

16. Huo H, Li T, Zhang L (2013) pKa determination of oxysophocarpine by reversed - phase high performance liquid chromatography. Springerplus 2: 270.

17. Jorge Ramos (2014) UV-Vis Spectrometry, pKa of a dye. 1-7.

18. Chemagination (2009) How to measure pKa by UV-vis spectrophotometry

19. Martínez CHR, Dardonville C (2013) Rapid determination of ionization constants (pKa) by UV spectroscopy using 96 -well microtiter plates. ACS Med Chem Lett 4: 142-145. 